\title{
Non-degenerate squarks from flavored gauge mediation
}

\author{
Iftah Galon, ${ }^{a}$ Gilad Perez ${ }^{b, c}$ and Yael Shadmi ${ }^{a}$ \\ ${ }^{a}$ Physics Department, Technion, Israel Institute of Technology, \\ Haifa 32000, Israel \\ ${ }^{b}$ CERN Theory Division, $C H-1211$, \\ Geneva 23, Switzerland \\ ${ }^{c}$ Department of Particle Physics and Astrophysics, \\ and Weizmann Institute of Science, Rehovot 76100, Israel \\ E-mail: iftah@tx.technion.ac.il, gilad.perez@cern.ch, \\ yshadmi@physics.technion.ac.il
}

ABStract: We study the squark spectra of Flavored Gauge Mediation Models, in which messenger-matter superpotential couplings generate new, generation-dependent contributions to the squark masses. The new couplings are controlled by the same flavor symmetry that explains the fermion masses, leading to excellent alignment of the quark and squark mass matrices. This allows for large squark mass splittings consistent with all flavor bounds. In particular, second-generation squarks are often significantly lighter than the first-generation squarks. As squark production at the LHC is dominated by the up- and down-squarks and the efficiencies for squark searches increase with their masses, the charm and/or strange squark masses can be well below the current LHC bounds. At the same time, even with a single set of messengers, the models can generate large stop mixings which result in large loop contributions to the Higgs mass.

KEYWORds: Supersymmetry Phenomenology

ARXIV EPRINT: 1306.6631 


\section{Contents}

1 Introduction 1

2 Setup and general considerations $\quad 3$

3 Non-degenerate squarks $\quad 6$

$\begin{array}{ll}3.1 & \text { Flavor symmetry and fermion masses }\end{array}$

$\begin{array}{lll}3.2 & \text { Light charm- and strange-squarks } & 7\end{array}$

3.3 Heavy up- and down-squarks 8

3.4 A light right-handed charm squark and a heavy Higgs 9

4 Conclusions $\quad 10$

$\begin{array}{ll}\text { A Soft terms } & 11\end{array}$

\section{Introduction}

As ATLAS and CMS are excluding large portions of the parameter space of supersymmetric extensions of the standard model, it is important to ensure that superpartners are not escaping detection simply because current searches are optimized for specific spectra. One common assumption is that first- and second-generation squarks are degenerate. There are various viable schemes however for the mediation of supersymmetry breaking in which this assumption does not hold, leading to different and distinct collider signatures [1-7]. A particularly intriguing possibility is that second-generation squarks are significantly lighter than the current $\mathcal{O}(1.5 \mathrm{TeV})$ bounds [8-15] which are mainly sensitive to the "valence" squark masses and are optimized to look for heavy squarks [16]. In fact recasting existing analyses it was found in [16] that a single weak singlet of the second generation such as the charm or strange squark as light as $\mathcal{O}(400 \mathrm{GeV})$ is consistent with current direct searches. The mixing of such a state with the stops is also unconstrained at present and would weaken the bound on their masses [17, 18].

Non-degenerate $\mathrm{TeV}$-scale first- and second-generation squarks can be consistent with bounds on flavor-violating processes in alignment models [19, 20]. In these models, both the fermion and sfermion mass matrices are controlled by a broken flavor symmetry, so that they are approximately diagonal in the supersymmetry interaction basis. Specifically, the mass-squared matrices for the SU(2)-singlet up (down) squarks must be aligned with the up (down) singlet quark Yukawa matrices, while the doublet squark mass-squared matrix needs to be aligned with the down-quark Yukawa matrix. It was pointed out recently that, despite the considerable progress in constraining CP violation in $D-\bar{D}$ mixing (see [21]), alignment models can be consistent with all flavor constraints [22-24]. However, known examples of 
alignment are typically high-scale models, with supersymmetry-breaking mediated to the Minimal Supersymmetric Standard Model (MSSM) at scales close to the GUT scale. The gluino mass then induces a large universal contribution to the squark masses through the RGE evolution to the weak scale. Since this contribution makes up $80-85 \%$ to the squark masses, these models can only lead to modest non-degeneracies in the squark spectrum.

An alternative scenario which allows for sfermion non-degeneracy with flavor violation suppressed by alignment, is Flavored Gauge Mediation (FGM) [6]. FGM models are simple variations of Minimal Gauge Mediation models [25, 26], with superpotential couplings of the messengers to the matter fields. These couplings generate new, generation-dependent contributions to the soft masses, which are nonetheless consistent with flavor bounds, if the resulting contributions are aligned with the fermion matrices. This possibility can be realized in the context of flavor symmetries as in [19]. However, while in the models of [19], the flavor symmetry controls the soft-supersymmetry breaking terms, FGM models exhibit supersymmetric alignment, with the flavor symmetry controlling the new superpotential couplings. Thus, alignment can be achieved in these models even for a low supersymmetry breaking scale, for which RGE effects are small.

Gauge Mediated Supersymmetry Breaking (GMSB) models with messenger-matter couplings were originally studied in [27-33]. Unlike pure GMSB models [34-39], these models have A-terms at the messenger scale. In addition, the new contributions to the stop masses are negative in large parts of the parameter space. As a result, stop mixing can be significant in these models, which allows for a heavy Higgs with relatively light superpartners [40-50].

In section 2 we describe the structure of FGM models, and explain the qualitative behavior of the new contributions to the squark masses-squared. These contributions start at one-loop, but the one-loop results are sub-leading in the supersymmetry breaking. Thus these one-loop contributions dominate at low messenger scales, where the universal RGE contribution is smallest. Since the one-loop contributions are always negative, the masses are always driven lower in this part of the parameter space. At higher messenger scales, roughly above $10^{7} \mathrm{GeV}$, the two-loop contributions dominate. These are negative for small messenger-matter couplings, and change sign for couplings of order unity. We also collect in this section the relevant flavor bounds, and discuss their implications for our models.

In section 3 we give several examples, for a single set of messengers with up-type messenger couplings. The models are then characterized by the charge assignments of the messengers under the flavor symmetry. These lead to diverse and interesting spectra, including examples in which the charm and strange squarks are significantly lighter than the GMSB predictions, or conversely, some of the up and down quarks are heavier than the GMSB predictions.

Interestingly, even with a single set of messengers, the new couplings can generate large contributions to the stop masses and A-terms together with large and negative contributions to the charm squark. Thus, these models can accommodate a $126 \mathrm{GeV}$ Higgs mass with a non-degenerate squark spectrum.

The relevant expressions for the soft terms are collected in appendix A. 


\begin{tabular}{|c|c|c|c|c|c|}
\hline Superfield & $X$ & $D_{1}$ & $\bar{D}_{1}$ & $D_{I \neq 1}, \bar{D}_{I \neq 1}$ & $T_{I}, \bar{T}_{I}$ \\
\hline$Z_{3}$ & 1 & -1 & 0 & 1 & 1 \\
\hline
\end{tabular}

Table 1. $Z_{3}$ charge assignments.

\section{Setup and general considerations}

In Flavored Gauge Mediation Models [6], the messenger fields have superpotential couplings to the MSSM matter fields, leading to new, generation dependent contributions to the soft terms. The models below are based on minimal GMSB models [25, 26], with a single supersymmetry-breaking spurion

$$
\langle X\rangle=M+F \theta^{2},
$$

and $N_{5}$ pairs of messengers transforming as $5+\overline{5}$ of SU(5). We use capital letters to denote the messenger fields, with $D$ transforming as $H_{D}, \bar{D}$ transforming as $H_{U}$, and $T$ and $\bar{T}$ denoting the corresponding triplet messengers.

We will only consider up-type messenger couplings, with the superpotential given by,

$$
W=X\left(\bar{T}_{I} T_{I}+\bar{D}_{I} D_{I}\right)+H_{U} q Y_{U} u^{c}+H_{D} q Y_{D} d^{c}+H_{D} l Y_{L} e^{c}+\bar{D} q y_{U} u^{c} .
$$

Here $q, u^{c}, d^{c}, l, e^{c}$ are the MSSM matter superfields, $Y_{U}, Y_{D}, Y_{L}$ are the MSSM Yukawa matrices, $y_{U}$ are the up-type messenger-matter Yukawa matrices, and $I=1, \ldots, N_{5}$ runs over the messenger pairs. $\bar{D} \equiv \bar{D}_{1}$ denotes the messenger field with superpotential couplings to matter, and similarly $D \equiv D_{1}$.

Note that we have chosen the $D$ and $\bar{D}$ messengers to have the same R-parity as the Higgses. There are different choices of symmetries which result in the structure of eq. (2.2), and forbid generic Higgs couplings to the messengers. One example is the standard U(1) Peccei-Quinn symmetry that forbids the mu-term, with for example, the Higgses carrying charge 2 and the matter fields carrying charge -1 each. Since we want $\bar{D}$ couplings to the matter fields, $\bar{D}$ must also carry charge 2 , with $D$ carrying charge -2 . Just as in minimal GMSB models, this symmetry still allows for explicit messenger mass terms. To forbid these as well, one can employ instead a U(1) or $Z_{3}$ symmetry, with the messengers transforming as in table 1 . In the following we will additionally impose a flavor symmetry with different $\bar{D}$ and $H_{U}$ charges. While these charges typically forbid the superpotential coupling $X D H_{U}$, there is always $\bar{D}-H_{U}$ mixing from the Kähler potential,

$$
K \supset \epsilon \bar{D}^{\dagger} H_{U}+\text { h.c. }
$$

where $\epsilon$ is given by a power of the spurion field which breaks the flavor symmetry. We can always redefine the fields to obtain a canonical Kähler potential, and subsequently identify the combination that couples to $X$ as the messenger $\bar{D}$, and the orthogonal combination as $H_{U} \cdot{ }^{1}$ As a result, the coupling $y_{U}$ is modified as,

$$
y_{U} \rightarrow y_{U}+\epsilon Y_{U} .
$$

\footnotetext{
${ }^{1}$ This last step involves a rotation of the canonically normalized fields.
} 
This modification will have very small effects in the models below. ${ }^{2}$

The messenger-matter couplings $y_{U}$ generate new contributions to the soft masses. These contributions were calculated for the 3 -generation case in [47] (see also $[49,50]$ ) and are collected in appendix A. The new contributions have a few noteworthy features. First, the new couplings generate A-terms at one-loop. If these have large 33 entries, the MSSM lower bound on the stop masses required for a $126 \mathrm{GeV}$ Higgs can be significantly relaxed [47]. These $A$ terms will have very small entries for the first and second generations in all of our models. Second, the scalar masses-squared receive 2-loop contributions from the new couplings, which, just like the pure GMSB contributions, appear at leading order in the supersymmetry breaking, $\mathcal{O}\left(F^{2} / M^{2}\right)$. These involve $y^{4}$ terms, mixed gauge- $y^{2}$ terms and mixed $y^{2}-Y^{2}$ terms. The latter can have either sign, and their effects on the first and second generation squarks are usually negligible. On the other hand, when the matrix $y_{U}$ has a single dominant entry, as will be the case in all our examples, the former terms have definite signs: the $y^{4}$ contributions are positive and the gauge- $y^{2}$ contributions are negative. In addition, there are one-loop contributions at $\mathcal{O}\left(F^{4} / M^{6}\right)$, which are always negative. Since the ratio of the one-loop to the two-loop contribution scales as $F^{2} / M^{4}$, and $F / M$ gives the overall scale of the soft masses, the one-loop contributions are only important at low messenger scales.

It is instructive to examine the new contributions in the simple case that only the 11 or 22 entry of $y_{u}$ is non-zero. This will indeed be the case in most of our examples below. ${ }^{3}$ Taking for example

$$
y_{u i j}=y \delta_{i 2} \delta_{j 2},
$$

with all other entries zero or negligible, the only change in the squark mass matrices is

$$
\left(\delta m_{q}^{2}\right)_{22}=\frac{1}{2}\left(\delta m_{u}^{2}\right)_{22} \equiv \delta m^{2},
$$

with, to leading order in $F / M^{2}$,

$$
\delta m^{2} \sim-\frac{1}{(4 \pi)^{2}} \frac{1}{6}|y|^{2} \frac{F^{4}}{M^{6}}+\frac{1}{(4 \pi)^{4}}\left(6|y|^{2}-G_{y}\right)|y|^{2} \frac{F^{2}}{M^{2}},
$$

where

$$
G_{y} \equiv \frac{16}{3} g_{3}^{2}+3 g_{2}^{2}+\frac{13}{15} g_{1}^{2} .
$$

In figure 1, we plot the relative change in the squark mass squared at the messenger scale,

$$
r_{m^{2}}(M) \equiv \frac{\delta m^{2}}{m_{q, \mathrm{GMSB}}^{2}},
$$

where $m_{q, \mathrm{GMSB}}^{2}$ is the pure GMSB contribution at different messenger scales. For low

\footnotetext{
${ }^{2}$ Note that the shift in $y_{U}$ is proportional to the matrix $Y_{U}$ and is therefore an MFV effect. If the superpotential term $X D H_{U}$ is allowed, it has the same parametric suppression $\epsilon$, and leads to a modification of $Y_{U}$ as well: $Y_{U} \rightarrow Y_{U}+\epsilon y_{U}$.

${ }^{3}$ In fact, because the first- and second-generation Yukawas are negligible, the relevant quantities are $y_{U} y_{U}^{\dagger}$ and $y_{U}^{\dagger} y_{U}$ (see eq. (A.5)), and all we need to assume here is that these matrices have a single entry on the diagonal.
} 


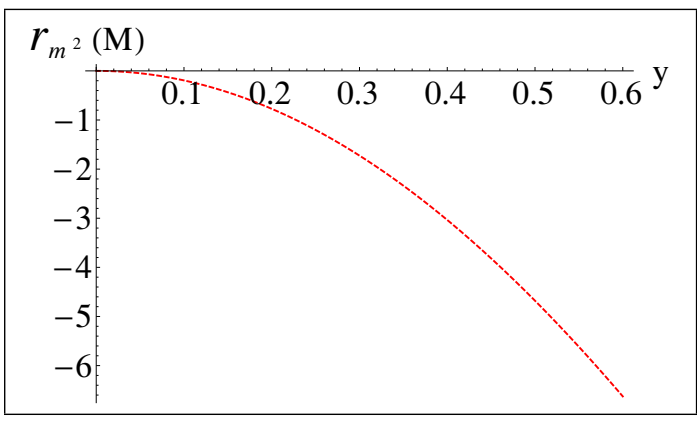

(a)

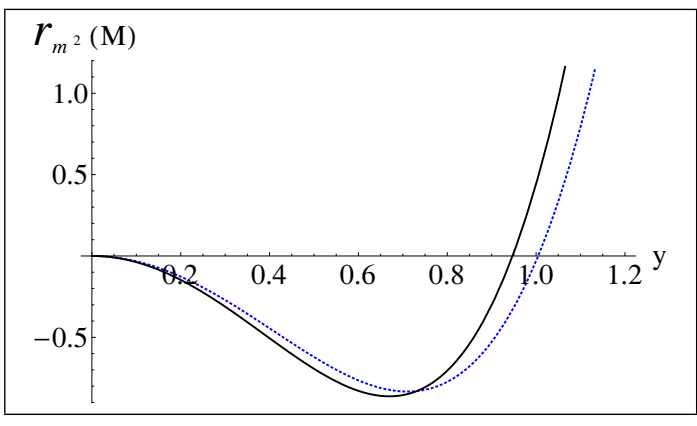

(b)

Figure 1. The ratio of the new contribution to the GMSB contribution, $r_{m^{2}}$, with $F / M=$ $8 \cdot 10^{4} \mathrm{GeV}$ and $N_{5}=1$, for three different values of the messenger scale $M$. In $1 \mathrm{a}$. we show the 1-loop contribution for $M=10^{5} \mathrm{GeV}$. In 1b. we show the 2-loop contribution for $M=10^{6} \mathrm{GeV}$ (dotted, blue), and $M=10^{8} \mathrm{GeV}$ (solid,black).

messenger scales (figure 1(a)), the negative one-loop contribution dominates, and the shift in the squark mass squared is sizeable even for low values of $y$. Thus the most interesting range of $y$ 's at these low scales is around 0.2 or so, which is naturally obtained with a single Cabibbo suppression. For larger messenger scales (figure 1(b)), the new contribution changes sign near $y=1$, so that there are two qualitatively different regions. For small $y$ 's, say 0.2 to 0.8 , the new contribution is negative and the relative mass splitting $r_{m^{2}}$ varies roughly between -0.5 to -1 . Since our flavor spurion will be roughly 0.2 , there is of course some ambiguity as to whether values of $y$ near 0.8 should be thought of as one or zero powers of the spurion. For $y>1$, the new contribution grows very fast, but such high values of the coupling are undesirable anyway from the point of view of perturbativity.

For an arbitrary coupling matrix $y_{U}$, the new contributions would lead to severe SUSY flavor and CP problems. However, as stressed in [6], any flavor theory that controls the Standard Model (SM) Yukawas will necessarily generate a non-trivial structure of the new couplings. In particular, if fermion masses are explained by a flavor symmetry, the new couplings will be determined (up to order one coefficients) once the flavor charges of the messengers are specified. Since we are mainly interested in large effects in the first and second generation squarks, our examples will be based on different flavor charges for $\bar{D}$ and $H_{U}$, such that $y_{U}$ has large entries in the first- and second-generation.

For convenience, we collect here the relevant bounds on flavor-violation involving the first two generations and $b \rightarrow s$ transitions from [51]. ${ }^{4}$ Since we are interested in LHC phenomenology, it is useful to work in the squark mass basis, and express the constrained quantities in terms of the squark masses, $m_{q_{i}}$, and the quark-squark-gluino couplings $K_{i j}$. The constrained quantities are then,

$$
\left(\delta_{i j}^{q}\right)_{M M}=\frac{\Delta \tilde{m}_{q_{j} q_{i}}^{2}}{\tilde{m}_{q}^{2}}\left(K_{M}^{q}\right)_{i j}\left(K_{M}^{q}\right)_{j j}^{*}
$$

\footnotetext{
${ }^{4}$ The bounds quoted here are a bit stronger compared to [51] as we assume order one CP-violating phases, as is generically expected in our framework. Furthermore, the bounds related to 23 mixing are taken to be $\sim 3$ times stronger compared to [51] due to the recent LHCb constraints on CP violation in $B_{s}$ mixing [52].
} 


\begin{tabular}{|cc|ccc|}
\hline$q$ & $i j$ & $\left|\left(\delta_{i j}^{q}\right)_{M M}\right|$ & $\sqrt{\operatorname{Im}\left(\delta_{i j}^{q}\right)_{M M}^{2}}$ & $\sqrt{\operatorname{Im}\left(\left(\delta_{i j}^{q}\right)_{L L}\left(\delta_{i j}^{q}\right)_{R R}\right)}$ \\
\hline$d$ & 12 & 0.07 & 0.01 & 0.0005 \\
$u$ & 12 & 0.1 & 0.05 & 0.003 \\
$d$ & 23 & 0.6 & 0.2 & 0.07 \\
\hline
\end{tabular}

Table 2. The upper bounds on $\left(\delta_{i j}^{q}\right)_{M M}$, taken from [51], but assuming order-one phases, for $m_{\tilde{q}}=1 \mathrm{TeV}$ and $m_{\tilde{g}} / m_{\tilde{q}}=1$.

where $\Delta \tilde{m}_{q_{j} q_{i}}^{2}=m_{q_{j}}^{2}-m_{q_{i}}^{2}$, and $\tilde{m}_{q}^{2}$ is the average of the relevant squark masses-squared of the same type. The experimental constraints on the $\delta$ 's as derived in [51] are summarized in table 2. These bounds should be viewed as rough estimates only, and will vary by order-1 numbers over the parameter space of our models. In particular, while the values in table 2 were derived for common squark and gluino masses, the gluino and different squark species have different masses in our models with values given predominantly by the GMSB contributions.

The most severe constraint is on the product of the LL and RR 1-2 mass splitting. Since the up LL and RR mass splittings are typically of the same order of magnitude in our models, a strong suppression of the 1-2 mixings is needed in order to allow for large mass differences. This can be achieved with a $\mathrm{U}(1) \times \mathrm{U}(1)$ flavor symmetry, which can lead to a high level of down alignment. Since the relative mass shift $r_{m^{2}}$ is suppressed by $N_{5}$, and since the gluino mass generates a universal contribution to the squark masses through the running, and furthermore, the gluino to squark mass ratio scales as $\sqrt{N_{5}}$, larger mass differences are obtained for $N_{5}=1$ and low messenger scales.

\section{Non-degenerate squarks}

\subsection{Flavor symmetry and fermion masses}

As explained above, in order to achieve sufficient suppression of the 1-2 mixing, we take the flavor symmetry to be $\mathrm{U}(1) \times \mathrm{U}(1)$, with each $\mathrm{U}(1)$ broken by a spurion $\lambda \sim 0.2$ of charge -1 . Each Yukawa matrix element is then either suppressed by the appropriate power of $\lambda$, or vanishes due to holomorphy. Since the superpotential can only depend on the spurion $\lambda$, and not $\lambda^{\dagger}$, superpotential terms of negative total $\mathrm{U}(1)$ charge cannot appear [19].

Following [20] we assign the $\mathrm{U}(1) \times \mathrm{U}(1)$ charges

$$
\begin{array}{lll}
Q_{1}(6,-3), & Q_{2}(2,0), & Q_{3}(0,0) \\
u_{1}^{c}(-6,9), & u_{2}^{c}(-2,3), & u_{3}^{c}(0,0) \\
d_{1}^{c}(-6,9), & d_{2}^{c}(2,0), & d_{3}^{c}(2,0)
\end{array}
$$

for the matter fields. The $H_{U}, H_{D}$ charges can always be chosen as zero [20]. The resulting SM Yukawas are

$$
Y_{U} \sim\left(\begin{array}{ccc}
\lambda^{6} & \lambda^{4} & 0 \\
0 & \lambda^{3} & \lambda^{2} \\
0 & 0 & 1
\end{array}\right), Y_{D} \sim\left(\begin{array}{ccc}
\lambda^{6} & 0 & 0 \\
0 & \lambda^{4} & \lambda^{4} \\
0 & \lambda^{2} & \lambda^{2}
\end{array}\right)
$$


where here and throughout the paper, the different entries of these matrices are known only up to order-one coefficients. These lead to the fermion mixing matrices,

$$
\begin{array}{rlrl}
V_{L}^{u} & \sim\left(\begin{array}{ccc}
1 & \lambda & 0 \\
\lambda & 1 & \lambda^{2} \\
0 & \lambda^{2} & 1
\end{array}\right), & V_{L}^{d} \sim\left(\begin{array}{ccc}
1 & 0 & 0 \\
0 & 1 & \lambda^{2} \\
0 & \lambda^{2} & 1
\end{array}\right), \\
V_{R}^{u} \sim\left(\begin{array}{ccc}
1 & \lambda^{4} & 0 \\
\lambda^{4} & 1 & \lambda^{5} \\
0 & \lambda^{5} & 1
\end{array}\right), & V_{R}^{d} \sim\left(\begin{array}{lll}
1 & 0 & 0 \\
0 & 1 & 1 \\
0 & 1 & 1
\end{array}\right) .
\end{array}
$$

The squark mass matrices are determined by the $\mathrm{U}(1) \times \mathrm{U}(1)$ flavor charges of the messengers. We choose these as

$$
D(-n, m) \quad \bar{D}(n,-m)
$$

where $n, m$ are integers. The different examples below correspond to different choices of $n$ and $m$. For $m>0$, the resulting $y_{U}$ coupling will have large entries in the 1-2 block. Furthermore, for $n>0$, the superpotential coupling $X D H_{U}$ is forbidden. Kähler mixing of $H_{U}$ and $\bar{D}$ is suppressed by $|\lambda|^{n+m}$.

\subsection{Light charm- and strange-squarks}

Choosing $n=1$ and $m=3$ we have

$$
y_{u}=\left(\begin{array}{ccc}
\lambda^{4} & 0 & 0 \\
0 & c_{22} \lambda & 0 \\
0 & 0 & 0
\end{array}\right)
$$

where the zeros follow from holomorphy as described above, and we explicitly displayed the order-one coefficient $c_{22}$ in the 22 entry. This model is then precisely of the type eq. (2.5). The 22 entries of the LL and RR up squark mass-squared matrices are modified as in eqs. (2.6) and (2.7), with $y=c_{22} \lambda$. All other entries of the up mass matrices, as well as the down mass matrices, remain virtually unmodified. For $c_{22} \sim 1, \delta m^{2}$ can be large, so that the $\mathrm{L}$ and $\mathrm{R}$ charm squark masses, as well as the L strange squark mass, are shifted to lower values. While we loosely refer to the squark mass eigenstates as "up", "charm" etc., this is of course not quite accurate, since the fermion mixing matrices of eq. (3.3) introduce some inter-generation mixings. Since the R down squark masses are unaltered, the only potentially observable effect is the $\mathcal{O}(\lambda) 12$ mixing in the L up sector. Here and in the following we continue to refer to the squark eigenstates by their dominant flavor component whenever the remaining flavor components are indeed very small.

The A-terms (in the flavor basis) are given by

$$
\delta A_{u}^{*} \sim-\frac{1}{16 \pi^{2}}\left(\begin{array}{ccc}
0 & \lambda^{6} & 0 \\
0 & \lambda^{5} & \lambda^{4} \\
0 & 0 & 0
\end{array}\right) \frac{F}{M}, \quad \delta A_{d}^{*} \sim-\frac{1}{16 \pi^{2}}\left(\begin{array}{ccc}
0 & 0 & 0 \\
0 & \lambda^{6} & \lambda^{6} \\
0 & 0 & 0
\end{array}\right) \frac{F}{M},
$$

and can therefore be neglected. ${ }^{5}$

\footnotetext{
${ }^{5}$ Furthermore, because the matrix $y_{U}$ has a single entry, which enters in its absolute value squared, these A terms do not contain any new phases on top of the SM Yukawas.
} 
While the $D$ and $\bar{D}$ charges forbid the superpotential term $X D H_{U}$, they do allow $\bar{D}-H_{U}$ Kähler mixing proportional to $\lambda^{4}$. As explained in section 2, the effect of this mixing is to modify the new coupling as

$$
y_{U} \rightarrow y_{U}+\lambda^{4} Y_{U}
$$

It is easy to see that this leads to negligible effects in all entries of $y_{u}$ and the resulting soft terms.

Flavor constraints are satisfied in this model even for large mass splittings thanks to the precise alignment of the down sector, which essentially eliminates any $\mathrm{CP}$ violating contributions. The only non-negligible $\delta$ 's are given by,

$$
\begin{aligned}
& \left(\delta_{12}^{u}\right)_{L L} \sim \lambda r_{m^{2}}, \quad\left(\delta_{23}^{u}\right)_{L L} \sim \lambda^{2} r_{m^{2}}, \quad\left(\delta_{23}^{d}\right)_{L L} \sim \lambda^{2} r_{m^{2}}, \\
& \left(\delta_{12}^{u}\right)_{R R} \sim \lambda^{4} r_{m^{2}}, \quad\left(\delta_{23}^{u}\right)_{R R} \sim \lambda^{5} r_{m^{2}}, \\
& \left(\delta_{12}^{u}\right)_{L R} \propto \lambda^{6}, \quad\left(\delta_{13}^{u}\right)_{L R} \propto \lambda^{5}, \quad\left(\delta_{23}^{u}\right)_{L R} \propto \lambda^{4} .
\end{aligned}
$$

Here $r_{m^{2}}$ is the relative mass splitting as defined in eq. (2.7) but evaluated at low energies. As the masses are evolved down to the TeV scale, $r_{m^{2}}$ decreases, due to the universal gaugino contribution. The most dramatic effects are therefore obtained for low messenger scales, and $N_{5}=1$. Thus for example, for $F / M=200 \mathrm{TeV}, M=500 \mathrm{TeV}$ and $\tan \beta=5$, starting with $r_{m^{2}}(M)=-1$, all the squark masses are around $2 \mathrm{TeV}$, the gluino mass is near 1.5 TeV, but the R-handed charm squark is at $870 \mathrm{GeV}$. Taking instead $F / M=150 \mathrm{TeV}$ and $M=400 \mathrm{TeV}$ the gluino is at $1.2 \mathrm{TeV}$, the R-handed charm squark at $670 \mathrm{GeV}$, while the remaining squarks are around $1.6 \mathrm{TeV}$.

To summarize, in large parts of the parameter space of this model (given essentially by the coefficient $c_{22}$ ), the two charm squarks and the left-handed strange squark are significantly lighter than the up and down squarks, with flavor constraints satisfied by virtue of the precise down alignment. There is however no effect on the Higgs mass in this model, since the couplings of eq. (3.6) vanish for the third generation.

\subsection{Heavy up- and down-squarks}

Taking $n=0$ and $m=6$ we again reproduce the coupling matrix $y_{U}$ of eq. (2.5), but now with a nonzero 11 entry,

$$
\left(y_{U}\right)_{i j}=y \delta_{1 i} \delta_{1 j}
$$

where $y$ is order-1. In the flavor basis, the messenger contributions to the the soft masses are again as in eq. (2.6)

$$
\begin{aligned}
\left(\delta \tilde{m}_{q}^{2}\right)_{11} & =\frac{1}{2}\left(\delta \tilde{m}_{u_{R}}^{2}\right)_{11}=\delta m^{2}, \\
\delta \tilde{m}_{d_{R}}^{2} & \sim 0
\end{aligned}
$$

Since the valence squarks are shifted here, this model is mainly interesting when $\delta m^{2}$ is positive. This implies that a. the one-loop contribution should be subdominant, which is the case for messenger scales above roughly $10^{7} \mathrm{GeV}$, and b. that $y$ should be close to 1 
(see figure 1(b)). Of course, for high messenger scales, RGE effects will significantly reduce the relative mass splittings.

The A-terms in this basis are given by

$$
\delta A_{u}^{*} \sim-\frac{|y|^{2}}{16 \pi^{2}}\left(\begin{array}{ccc}
3 \lambda^{6} & \lambda^{4} & 0 \\
0 & 0 & 0 \\
0 & 0 & 0
\end{array}\right) \frac{F}{M}, \quad \delta A_{d}^{*} \sim-\frac{|y|^{2}}{16 \pi^{2}}\left(\begin{array}{ccc}
\lambda^{6} & 0 & 0 \\
0 & 0 & 0 \\
0 & 0 & 0
\end{array}\right) \frac{F}{M} .
$$

All entries in the A-terms are very small.

Thus, both the left-handed and right-handed squarks of the first generation are split in mass from the second generation, with the relative mass splitting $\delta m^{2} / m^{2}$ again as in eq. (2.7). For $y \gtrsim 1$ the two up squarks and the left down squark are therefore heavier than the remaining squarks and the relative mass splitting can be a large effect. The strict bounds on flavor violation in the down sector are however satisfied thanks to the excellent alignment of the down squark and quark mass matrices. Since the squark mass matrix is diagonal in the flavor symmetry basis, the mixings arise only from the fermion Yukawa matrices of eq. (3.3). The only non-negligible $\delta$ s are therefore

$$
\left(\delta_{12}^{u}\right)_{L L} \sim \lambda r_{m^{2}}, \quad\left(\delta_{12}^{u}\right)_{R R} \sim \lambda^{4} r_{m^{2}}, \quad\left(\delta_{12}^{u}\right)_{L R} \propto \lambda^{4},
$$

and all of these are below the experimental bounds. As in the previous model, the effects of the $\bar{D}-H_{U}$ kinetic mixing in the Kähler potential are negligible as they are proportional to $\lambda^{6}$.

In this example too, there are no new couplings of the messengers to the third generation squarks, and the Higgs mass is therefore unaffected. To obtain a heavy Higgs, some modification of the Higgs sector is required.

\subsection{A light right-handed charm squark and a heavy Higgs}

Choosing $n=2$ and $m=3$

$$
y_{u}=\left(\begin{array}{ccc}
\lambda^{5} & 0 & 0 \\
0 & \lambda^{2} & 0 \\
0 & y & 0
\end{array}\right)
$$

where $y=\mathcal{O}(1)$, and as usual we do not display the $\mathcal{O}(1)$ prefactors of the powers of $\lambda$ in the remaining terms.

Again we find that the right handed charm squark mass is shifted,

$$
\delta\left(\tilde{m}_{u_{R}}^{2}\right)_{22} \sim-\frac{1}{3} \frac{1}{(4 \pi)^{2}}|y|^{2} \frac{F^{4}}{M^{6}}+\frac{1}{(4 \pi)^{4}} 2\left(\left(6+\left|\left(Y_{u}\right)_{33}\right|^{2}\right)|y|^{2}-G_{y}\right)|y|^{2} \frac{F^{2}}{M^{2}},
$$

and this shift is negative in large regions of the parameter space. ${ }^{6}$

At the same time however, because of the off-diagonal entry of $y_{U}$, there is also a large negative contribution to the right-handed stop mass,

$$
\delta\left(\tilde{m}_{u_{R}}^{2}\right)_{33} \sim-2|y|^{2}\left|\left(Y_{u}\right)_{33}\right|^{2} \frac{F^{2}}{M^{2}} .
$$

\footnotetext{
${ }^{6}$ The right-handed down squark matrix has $\mathcal{O}\left(\lambda^{4}\right)$ entries in the $2-3$ block, but these do not produce any significant effects.
} 
Note that this contribution is qualitatively different than those commonly considered in studies focused on raising the Higgs mass, which assume only a 33 entry in $y_{U}$ [4044, 47-50]. In particular, there is no positive $y_{U}^{4}$ contribution, or mixed gauge-Yukawa contribution.

As for the doublet squarks, these involve the combination

$$
y_{U} y_{U}^{\dagger} \sim\left(\begin{array}{ccc}
0 & 0 & 0 \\
0 & \lambda^{4} & \lambda^{2} \\
0 & \lambda^{2} & |y|^{2}
\end{array}\right)
$$

which, because the 3 rd column of $y_{U}$ vanishes, has only one non-zero eigenvalue. Thus only the masses of the third generation doublet squarks are affected, with

$$
\left(\delta \tilde{m}^{2}\right)_{\tilde{t}_{L}}=\left(\delta \tilde{m}^{2}\right)_{\tilde{b}_{L}} \sim-\frac{1}{6} \frac{1}{(4 \pi)^{2}}|y|^{2} \frac{F^{4}}{M^{6}}+\frac{1}{(4 \pi)^{4}}\left(6|y|^{2}-G_{y}\right)|y|^{2} \frac{F^{2}}{M^{2}},
$$

with a $2-3$ mixing of order $\lambda^{2}$.

The A-terms in the flavor basis are given by

$$
\delta A_{u}^{*} \sim-\frac{1}{16 \pi^{2}}\left(\begin{array}{ccc}
0 & \lambda^{4} & 0 \\
0 & \lambda^{3} & \lambda^{2} \\
0 & \lambda^{5} & |y|^{2}\left(Y_{u}\right)_{33}
\end{array}\right) \frac{F}{M}, \quad \delta A_{d}^{*} \sim-\frac{1}{16 \pi^{2}}\left(\begin{array}{ccc}
0 & 0 & 0 \\
0 & \lambda^{4} & \lambda^{4} \\
0 & \lambda^{2} & \lambda^{2}
\end{array}\right) \frac{F}{M},
$$

Thus, there is an order- 1 stop A term. Coupled with the new negative contributions to the stop masses, this leads to a large stop mixing which enhances the loop contributions to the Higgs mass. Therefore, even with a single messenger pair, the off-diagonal structure of the coupling $y_{U}$ results in a single light charm squark (the R-handed one), as well as a large Higgs mass.

The only non-negligible $\delta$ s are

$$
\begin{aligned}
& \left(\delta_{12}^{u}\right)_{L L} \sim \lambda^{5}, \quad\left(\delta_{13}^{u}\right)_{L L} \sim \lambda^{3}, \quad\left(\delta_{23}^{u}\right)_{L L} \sim \lambda^{2}, \quad\left(\delta_{23}^{d}\right)_{L L} \sim \lambda^{2}, \quad\left(\delta_{12}^{u}\right)_{R R} \sim \lambda^{4}, \\
& \left(\delta_{23}^{u}\right)_{R R} \sim \lambda^{5}, \quad\left(\delta_{12}^{u}\right)_{L R} \propto \lambda^{4}, \quad\left(\delta_{13}^{u}\right)_{L R} \propto \lambda^{3}, \quad\left(\delta_{23}^{u}\right)_{L R} \propto \lambda^{2}, \quad\left(\delta_{23}^{d}\right)_{L R} \propto \lambda^{5} .
\end{aligned}
$$

\section{Conclusions}

Flavored Gauge Mediation models provide a fully calculable framework for generating the MSSM soft terms, with the soft terms generated by the SM gauge interactions and superpotential couplings of the messenger and matter superfields. As we have seen, they allow for large mass splittings between the squarks, with flavor constraints satisfied by alignment. In particular, given that it is the superpotential couplings that are controlled by the flavor symmetry, this alignment can be achieved even at low messenger scales, reducing the large logs and the resulting fine-tuning typical of high-scale models.

The models lead to very interesting spectra from the point of view of direct searches at the LHC. In particular, charm squarks can be significantly lighter than the up and down squarks, and below current limits on the squark mass. For a small number of messenger 
pairs, the charm squark may be the only squark lighter than the gluino, so that the gluino predominantly decays into on-shell charm squarks. In addition, charm squarks generically have an $\mathcal{O}(\lambda)$ mixing with the up quark, which is typical of alignment models [16]. If charm tagging becomes possible in future experiments, observing the different up and charm components of the light charm squarks would be extremely interesting.

Finally, since the new couplings generate messenger-scale A-terms, these models can lead both to large mass splittings among the first and second generation squarks, and to large contributions to the Higgs mass, even with a single messenger pair, and we showed one example of this type in section 3.4. In other examples, only the first and second generation squark masses are altered, so that some additional ingredients are needed in order to raise the Higgs mass. This can involve, for example, an additional messenger which predominantly couples to the stops, or an extended Higgs sector as in the NMSSM.

\section{Acknowledgments}

GP thanks Gian Giudice, Paride Paradisi and Giovanni Villadoro for useful discussions. The research of Y. Shadmi and I. Galon was supported by the Israel Science Foundation (ISF) under grant No. 1367/11, and by the United States-Israel Binational Science Foundation (BSF) under grant No. 2010221. IG is also supported by the 2013 Gutwirth fellowship. IG thanks the CERN TH division for their hospitality while this work was in progress. GP is supported by grants from GIF, IRG, ISF and Minerva.

\section{A Soft terms}

The pure GMSB contributions to the soft squared masses and A-terms are given by [25, 26]

$$
\begin{aligned}
\tilde{m}_{q}^{2} & =\frac{1}{(4 \pi)^{4}} 2 N_{5}\left(\frac{4}{3} g_{3}^{4}+\frac{3}{4} g_{2}^{4}+\frac{1}{60} g_{1}^{4}\right)\left|\frac{F}{M}\right|^{2} 1_{3 \times 3}, \\
\tilde{m}_{u_{R}}^{2} & =\frac{1}{(4 \pi)^{4}} 2 N_{5}\left(\frac{4}{3} g_{3}^{4}+g_{1}^{4} \frac{4}{15}\right)\left|\frac{F}{M}\right|^{2} 1_{3 \times 3}, \\
\tilde{m}_{d_{R}}^{2} & =\frac{1}{(4 \pi)^{4}} 2 N_{5}\left(\frac{4}{3} g_{3}^{4}+\frac{1}{15} g_{1}^{4}\right)\left|\frac{F}{M}\right|^{2} 1_{3 \times 3}, \\
A_{u} & =A_{d}=0 .
\end{aligned}
$$

where we have only shown the leading order terms in $F / M^{2}$. As is well known [53], the higher order corrections to these expressions are small.

In the presence of up type matter-messenger couplings [47] the squared-masses of squarks receive both 1-loop and 2-loop contributions given by

$$
\begin{aligned}
\delta \tilde{m}_{q}^{2}=-\frac{1}{(4 \pi)^{2}} \frac{1}{6}\left(y_{u} y_{u}^{\dagger}\right) \frac{F^{4}}{M^{6}} h(x) & \\
+\frac{1}{(4 \pi)^{4}}\{(3 & \left.\operatorname{Tr}\left(y_{u}^{\dagger} y_{u}\right)-\frac{16}{3} g_{3}^{2}-3 g_{2}^{2}-\frac{13}{15} g_{1}^{2}\right) y_{u} y_{u}^{\dagger} \\
+ & 3 y_{u} y_{u}^{\dagger} y_{u} y_{u}^{\dagger}+2 y_{u} Y_{u}^{\dagger} Y_{u} y_{u}^{\dagger}-2 Y_{u} y_{u}^{\dagger} y_{u} Y_{u}^{\dagger} \\
& \left.+y_{u} Y_{u}^{\dagger} \operatorname{Tr}\left(3 y_{u}^{\dagger} Y_{u}\right)+Y_{u} y_{u}^{\dagger} \operatorname{Tr}\left(3 Y_{u}^{\dagger} y_{u}\right)\right\}\left|\frac{F}{M}\right|^{2},
\end{aligned}
$$




$$
\begin{aligned}
\delta \tilde{m}_{u_{R}}^{2}= & -\frac{1}{(4 \pi)^{2}} \frac{1}{3}\left(y_{u}^{\dagger} y_{u}\right) \frac{F^{4}}{M^{6}} h(x) \\
& +\frac{1}{(4 \pi)^{4}}\left\{2\left(3 \operatorname{lr}\left(y_{u}^{\dagger} y_{u}\right)-\frac{16}{3} g_{3}^{2}-3 g_{2}^{2}-\frac{13}{15} g_{1}^{2}\right) y_{u}^{\dagger} y_{u}\right. \\
& +6 y_{u}^{\dagger} y_{u} y_{u}^{\dagger} y_{u}+2 y_{u}^{\dagger} Y_{u} Y_{u}^{\dagger} y_{u}+2 y_{u}^{\dagger} Y_{d} Y_{d}^{\dagger} y_{u}-2 Y_{u}^{\dagger} y_{u} y_{u}^{\dagger} Y_{u} \\
& \left.+2 y_{u}^{\dagger} Y_{u} T r\left(3 Y_{u}^{\dagger} y_{u}\right)+2 Y_{u}^{\dagger} y_{u} \operatorname{Tr}\left(3 y_{u}^{\dagger} Y_{u}\right)\right\}\left|\frac{F}{M}\right|^{2} \\
\delta \tilde{m}_{d_{R}}^{2}= & -\frac{1}{(4 \pi)^{4}} 2 Y_{d}^{\dagger} y_{u} y_{u}^{\dagger} Y_{d}\left|\frac{F}{M}\right|^{2} .
\end{aligned}
$$

where $x=\frac{F}{M^{2}}$ and [40]

$$
h(x)=-3 \frac{(2-x) \log (1-x)+(2+x) \log (1+x)}{x^{4}} \approx 1+\frac{4 x^{2}}{5}+\mathcal{O}\left(x^{4}\right)
$$

In addition, A-terms receive 1-loop contributions

$$
\begin{aligned}
A_{u}^{*} & =-\frac{1}{16 \pi^{2}}\left[\left(y_{u} y_{u}^{\dagger}\right) Y_{u}+2 Y_{u}\left(y_{u}^{\dagger} y_{u}\right)\right] \frac{F}{M}, \\
A_{d}^{*} & =-\frac{1}{16 \pi^{2}}\left[\left(y_{u} y_{u}^{\dagger}\right) Y_{d}\right] \frac{F}{M}, \\
A_{l}^{*} & =0 .
\end{aligned}
$$

Note that we have defined

$$
Y_{u}=Y_{U}^{*}, \quad Y_{d}=Y_{D}^{*}, \quad Y_{l}=Y_{L}^{*}, \quad y_{u}=y_{U}^{*}
$$

such that $Y_{u, d, l}$ coincide with the SM Yukawa matrices.

Open Access. This article is distributed under the terms of the Creative Commons Attribution License which permits any use, distribution and reproduction in any medium, provided the original author(s) and source are credited.

\section{References}

[1] J.L. Feng, C.G. Lester, Y. Nir and Y. Shadmi, The standard model and supersymmetric flavor puzzles at the Large Hadron Collider, Phys. Rev. D 77 (2008) 076002 [arXiv:0712.0674] [INSPIRE].

[2] G.D. Kribs, E. Poppitz and N. Weiner, Flavor in supersymmetry with an extended R-symmetry, Phys. Rev. D 78 (2008) 055010 [arXiv:0712.2039] [InSPIRE].

[3] Y. Nomura, M. Papucci and D. Stolarski, Flavorful supersymmetry from higher dimensions, JHEP 07 (2008) 055 [arXiv:0802.2582] [INSPIRE].

[4] Y. Nomura and D. Stolarski, Naturally Flavorful Supersymmetry at the LHC, Phys. Rev. D 78 (2008) 095011 [arXiv:0808.1380] [INSPIRE].

[5] J.L. Feng et al., Measuring slepton masses and mixings at the LHC, JHEP 01 (2010) 047 [arXiv:0910.1618] [INSPIRE]. 
[6] Y. Shadmi and P.Z. Szabo, Flavored gauge-mediation, JHEP 06 (2012) 124 [arXiv: 1103.0292] [INSPIRE].

[7] I. Galon and Y. Shadmi, Kinematic edges with flavor splitting and mixing, Phys. Rev. D 85 (2012) 015010 [arXiv:1108.2220] [INSPIRE].

[8] CMS collaboration, Search for supersymmetry in events with photons and missing energy, CMS-PAS-SUS-12-018 (2012).

[9] ATLAS collaboration, Search for supersymmetry in events with large missing transverse momentum, jets and at least one $\tau$ lepton in $21 \mathrm{fb}^{-1}$ of $\sqrt{\mathrm{s}}=8 \mathrm{TeV}$ proton-proton collision data with the ATLAS detector, ATLAS-CONF-2013-026 (2013).

[10] CMS collaboration, Search for supersymmetry in hadronic final states with missing transverse energy using the variables $\alpha_{T}$ and b-quark multiplicity in pp collisions at $\sqrt{s}=8$ TeV, arXiv: 1303.2985 [INSPIRE].

[11] ATLAS collaboration, Search for non-pointing photons in the diphoton and $E_{T}^{\text {miss }}$ final state in $\sqrt{s}=7$ TeV proton-proton collisions using the ATLAS detector, Phys. Rev. D 88 (2013) 012001 [arXiv: 1304.6310] [INSPIRE].

[12] ATLAS collaboration, Search for squarks and gluinos with the ATLAS detector in final states with jets and missing transverse momentum and $20.3 \mathrm{fb}^{-1}$ of $\sqrt{\mathrm{s}}=8 \mathrm{TeV}$ proton-proton collision data, ATLAS-CONF-2013-047 (2013).

[13] ATLAS collaboration, Search for new phenomena using final states with large jet multiplicities and missing transverse momentum with ATLAS in $20 \mathrm{fb}^{-1}$ of $\sqrt{\mathrm{s}}=8 \mathrm{TeV}$ proton-proton collisions, ATLAS-CONF-2013-054 (2013).

[14] ATLAS collaboration, Search for strong production of supersymmetric particles in final states with missing transverse momentum and at least three b-jets using $20.1 \mathrm{fb}^{-1}$ of pp collisions at $\sqrt{s}=8 \mathrm{TeV}$ with the ATLAS Detector., ATLAS-CONF-2013-061 (2013).

[15] ATLAS collaboration, Search for squarks and gluinos in events with isolated leptons, jets and missing transverse momentum at $\sqrt{s}=8 \mathrm{TeV}$ with the ATLAS detector, ATLAS-CONF-2013-062 (2013).

[16] R. Mahbubani, M. Papucci, G. Perez, J.T. Ruderman and A. Weiler, Light non-degenerate squarks at the LHC, Phys. Rev. Lett. 110 (2013) 151804 [arXiv:1212.3328] [INSPIRE].

[17] M. Blanke, G.F. Giudice, P. Paradisi, G. Perez and J. Zupan, Flavoured naturalness, JHEP 06 (2013) 022 [arXiv: 1302.7232] [INSPIRE].

[18] P. Agrawal and C. Frugiuele, Mixing stops at the LHC, arXiv:1304.3068 [INSPIRE].

[19] Y. Nir and N. Seiberg, Should squarks be degenerate?, Phys. Lett. B 309 (1993) 337 [hep-ph/9304307] [INSPIRE].

[20] M. Leurer, Y. Nir and N. Seiberg, Mass matrix models: the sequel, Nucl. Phys. B 420 (1994) 468 [hep-ph/9310320] [INSPIRE].

[21] LHCb collaboration, Observation of $D^{0}-\bar{D}^{0}$ oscillations, Phys. Rev. Lett. 110 (2013) 101802 [arXiv:1211.1230] [INSPIRE].

[22] K. Blum, Y. Grossman, Y. Nir and G. Perez, Combining $K^{0}-\bar{K}^{0}$ mixing and $D^{0}-\bar{D}^{0}$ mixing to constrain the flavor structure of new physics, Phys. Rev. Lett. 102 (2009) 211802 [arXiv: 0903.2118] [INSPIRE]. 
[23] O. Gedalia, J.F. Kamenik, Z. Ligeti and G. Perez, On the universality of CP-violation in $\Delta F=1$ processes, Phys. Lett. B 714 (2012) 55 [arXiv:1202.5038] [INSPIRE].

[24] A. Kadosh, P. Paradisi and G. Perez, Alignment models and charm CP violation, to appear.

[25] M. Dine, A.E. Nelson and Y. Shirman, Low-energy dynamical supersymmetry breaking simplified, Phys. Rev. D 51 (1995) 1362 [hep-ph/9408384] [InSPIRE].

[26] M. Dine, A.E. Nelson, Y. Nir and Y. Shirman, New tools for low-energy dynamical supersymmetry breaking, Phys. Rev. D 53 (1996) 2658 [hep-ph/9507378] [INSPIRE].

[27] G. Dvali, G. Giudice and A. Pomarol, The $\mu$ problem in theories with gauge mediated supersymmetry breaking, Nucl. Phys. B 478 (1996) 31 [hep-ph/9603238] [INSPIRE].

[28] M. Dine, Y. Nir and Y. Shirman, Variations on minimal gauge mediated supersymmetry breaking, Phys. Rev. D 55 (1997) 1501 [hep-ph/9607397] [INSPIRE].

[29] G. Giudice and R. Rattazzi, Extracting supersymmetry breaking effects from wave function renormalization, Nucl. Phys. B 511 (1998) 25 [hep-ph/9706540] [INSPIRE].

[30] Z. Chacko and E. Ponton, Yukawa deflected gauge mediation, Phys. Rev. D 66 (2002) 095004 [hep-ph/0112190] [INSPIRE].

[31] F. Joaquim and A. Rossi, Gauge and Yukawa mediated supersymmetry breaking in the triplet seesaw scenario, Phys. Rev. Lett. 97 (2006) 181801 [hep-ph/0604083] [INSPIRE].

[32] F. Joaquim and A. Rossi, Phenomenology of the triplet seesaw mechanism with Gauge and Yukawa mediation of SUSY breaking, Nucl. Phys. B 765 (2007) 71 [hep-ph/0607298] [INSPIRE].

[33] A. Brignole, F.R. Joaquim and A. Rossi, Beyond the standard seesaw: neutrino masses from Kähler operators and broken supersymmetry, JHEP 08 (2010) 133 [arXiv:1007.1942] [INSPIRE].

[34] M. Dine, W. Fischler and M. Srednicki, Supersymmetric technicolor, Nucl. Phys. B 189 (1981) 575 [INSPIRE].

[35] S. Dimopoulos and S. Raby, Supercolor, Nucl. Phys. B 192 (1981) 353 [inSPIRE].

[36] M. Dine and W. Fischler, A phenomenological model of particle physics based on supersymmetry, Phys. Lett. B 110 (1982) 227 [INSPIRE].

[37] M. Dine and M. Srednicki, More supersymmetric technicolor, Nucl. Phys. B 202 (1982) 238 [INSPIRE].

[38] L. Álvarez-Gaumé, M. Claudson and M.B. Wise, Low-energy supersymmetry, Nucl. Phys. B 207 (1982) 96 [INSPIRE].

[39] C.R. Nappi and B.A. Ovrut, Supersymmetric extension of the $\mathrm{SU}(3) \times \mathrm{SU}(2) \times \mathrm{U}(1)$ model, Phys. Lett. B 113 (1982) 175 [INSPIRE].

[40] J.L. Evans, M. Ibe and T.T. Yanagida, Relatively heavy Higgs boson in more generic gauge mediation, Phys. Lett. B 705 (2011) 342 [arXiv:1107.3006] [INSPIRE].

[41] J.L. Evans, M. Ibe and T.T. Yanagida, Probing extra matter in gauge mediation through the lightest Higgs boson mass, arXiv:1108.3437 [INSPIRE].

[42] J.L. Evans, M. Ibe, S. Shirai and T.T. Yanagida, A 125 GeV Higgs boson and muon $g-2$ in more generic gauge mediation, Phys. Rev. D 85 (2012) 095004 [arXiv:1201.2611] [INSPIRE]. 
[43] Z. Kang, T. Li, T. Liu, C. Tong and J.M. Yang, A heavy SM-like Higgs and a light stop from Yukawa-deflected gauge mediation, Phys. Rev. D 86 (2012) 095020 [arXiv:1203.2336] [INSPIRE].

[44] J.L. Evans, M. Ibe and T.T. Yanagida, The lightest Higgs boson mass in the MSSM with strongly interacting spectators, Phys. Rev. D 86 (2012) 015017 [arXiv:1204.6085] [INSPIRE].

[45] N. Craig, S. Knapen, D. Shih and Y. Zhao, A complete model of low-scale gauge mediation, JHEP 03 (2013) 154 [arXiv:1206.4086] [INSPIRE].

[46] A. Albaid and K. Babu, Higgs boson of mass 125 GeV in GMSB models with messenger-matter mixing, arXiv:1207.1014 [INSPIRE].

[47] M. Abdullah, I. Galon, Y. Shadmi and Y. Shirman, Flavored gauge mediation, a heavy Higgs and supersymmetric alignment, JHEP 06 (2013) 057 [arXiv: 1209.4904] [INSPIRE].

[48] N. Craig, S. Knapen and D. Shih, General messenger Higgs mediation, JHEP 08 (2013) 118 [arXiv: 1302.2642] [INSPIRE].

[49] J.A. Evans and D. Shih, Surveying extended GMSB models with $m h=125 \mathrm{GeV}$, JHEP 08 (2013) 093 [arXiv: 1303.0228] [INSPIRE].

[50] L. Calibbi, P. Paradisi and R. Ziegler, Gauge mediation beyond minimal flavor violation, JHEP 06 (2013) 052 [arXiv: 1304.1453] [INSPIRE].

[51] G. Isidori, Y. Nir and G. Perez, Flavor physics constraints for physics beyond the standard model, Ann. Rev. Nucl. Part. Sci. 60 (2010) 355 [arXiv:1002.0900] [InSPIRE].

[52] LHCb collaboration, First observation of $C P$ violation in the decays of $B_{s}^{0}$ mesons, Phys. Rev. Lett. 110 (2013) 221601 [arXiv:1304.6173] [INSPIRE].

[53] S.P. Martin, Generalized messengers of supersymmetry breaking and the sparticle mass spectrum, Phys. Rev. D 55 (1997) 3177 [hep-ph/9608224] [INSPIRE]. 